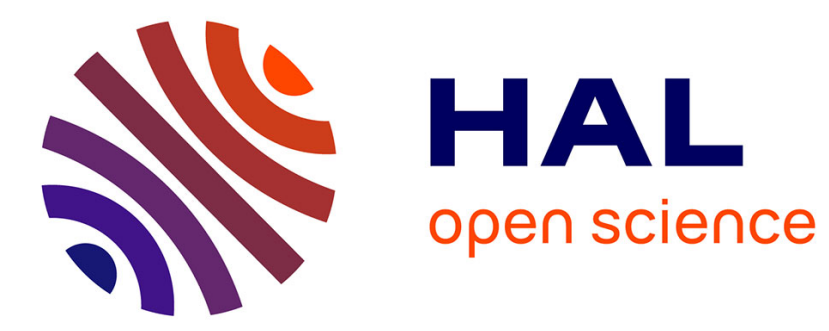

\title{
Undiscounted zero sum differential games with stopping times
}

\author{
Mabel M. Tidball
}

\section{To cite this version:}

Mabel M. Tidball. Undiscounted zero sum differential games with stopping times. [Research Report] RR-2294, INRIA. 1994. inria-00074379

\section{HAL Id: inria-00074379 \\ https://hal.inria.fr/inria-00074379}

Submitted on 24 May 2006

HAL is a multi-disciplinary open access archive for the deposit and dissemination of scientific research documents, whether they are published or not. The documents may come from teaching and research institutions in France or abroad, or from public or private research centers.
L'archive ouverte pluridisciplinaire HAL, est destinée au dépôt et à la diffusion de documents scientifiques de niveau recherche, publiés ou non, émanant des établissements d'enseignement et de recherche français ou étrangers, des laboratoires publics ou privés. 


\title{
Undiscounted zero sum differential games with stopping times
}

\author{
Mabel M. Tidball
}

$\mathbf{N}^{\circ} 2294$

Juin 1994

PROGRAMME 5

Traitement du signal,

automatique

et productique 



\title{
RIN RIA
}

\section{Undiscounted zero sum differential games with stopping times}

\author{
Mabel M. Tidball* \\ Programme 5 - Traitement du signal, automatique et productique \\ Projet MIAOU
}

Rapport de recherche $\mathrm{n}^{\circ} 2294$ - Juin 1994 - 21 pages

\begin{abstract}
We propose a discretization scheme for an undiscounted zero sum differential game with stopping times. The value function of the original problem satisfies an integral inequality of Isaacs type that we can discretize using finite difference or finite element techniques.

The fully discrete problem defines a stochastic game problem associated to the process, which may have, in general, multiple solutions. Among these solutions there exists one which is naturally associated with the value function of the original problem.

We completely characterize the set of solution and we describe a procedure to identify the desired solution. We present accelerated algorithms in order to compute efficiently the discrete solution.
\end{abstract}

(Résumé : tsvp)

*Facultad de Ciencias Exactas, Ingeniería y Agrimensura, Universidad Nacional de Rosario, Pellegrini 250, (2000) Rosario, Argentina. This work was partially done during a visit of the author at INRIA - Sophia Antipolis, France 


\section{JEUX DIFFERENTIELS A SOMME NULLE AVEC TEMPS D'ARRET SANS TAUX D'ACTUALISATION}

Résumé : On propose un schéma de discrétisation pour des jeux différentiels à somme nulle avec temps d'arrêt sans taux d'actualisation.

La fonction valeur du problème continu satisfait une inégalité de type Isaacs. On discrétise ces inégalités en utilisant des techniques de différences finies.

Le problème complètement discret définit un problème stochastique associé au procesus. Ce problème peut avoir plusieurs solutions. Néanmoins, une seule de ces solutions correspond à une approximation de la fonction valeur du problème continu. On caractérise l'ensemble des solutions et on décrit une procédure pour trouver la solution cherchée.

On présente un algorithme pour calculer efficacement la solution discrète. 


\section{Introduction}

We consider an undiscounted zero sum differential game problem with stopping times

. The same problem with discount rate $(\lambda>0)$ was study in [20] and a discretization procedure in [22].

For case $\lambda>0$ (see [20]) it is proved that the value function of the game is a Holder continuous function; but in the present case $(\lambda=0)$, we loose, in general, this regularity property. In spite of this discouraging result we can prove that the value function is always well defined and unique when the stopping costs verify a natural condition which correspond to a non trivial game; but even though we have regular data, the value function can be discontinuous. Works related with this type of phenomenon can be found in [1]. Problems without discount was also studied in [18] for ergodic control problems.

The aim of this work is to obtain a numerically computable discrete solution of this game and to precise when this solution represents the value function of our problem.

By the dynamic programming principle, we can prove that the value function satisfies an integral inequality system of Isaacs type (see [10], [11], [20], [22]). Since an exact solution of this type of problems is usually impossible, we must discretize the problem and to solved it numerically. In order to obtain a discrete problem we approximate, using finite element techniques (see [21]), these inequalities. The fully discrete problem that we obtain, may have, in general, multiple solutions. It defines a stochastic game problem with stopping time associated to the process.

Among these solutions, there exists one that is naturally associated with the value function of the original problem (remind that the solution of our problem is unique). In this work we completely characterize the set of solutions of the discrete problem and we describe a procedure to identify this particular discrete solution. We present, in order to compute it, accelerated algorithms based on the techniques given in [9] and [22].

We also present special cases where we can prove regularity properties of the value function and the existence of a unique solution of the discrete problem. In these cases we can prove the convergence, in the viscosity sense (see [8]), of the discrete solution to the value function of the original problem.

$\mathrm{RR} \mathrm{n}^{\circ} 2294$ 


\section{Description of the problem}

\subsection{The differential game problem}

In this differential game problem, the decision variables used by the players are the stopping times for the uncontrolled system. The evolution of the system is described by an ordinary differential equation of the form:

$$
\frac{d y(t)}{d t}=g(y(t)) ; \quad y(0)=x
$$

with $x \in \Omega \subset \mathbb{R}^{\mathrm{n}}$, an open bounded domain. We denote $\tau_{1}$ and $\tau_{2}$ the stopping times chosen by players 1 and 2 respectively. To those stopping times is associated a payoff $\mathbf{J}$ where $\mathbf{J}$ takes the form :

$$
\begin{array}{r}
J\left(x, \tau_{1}, \tau_{2}\right)=\varlimsup_{\tau \rightarrow \infty} \int_{0}^{\tau_{1} \wedge \tau_{2} \wedge \tau \wedge \theta} f(y(s)) d s+\phi_{1}\left(y\left(\tau_{1}\right)\right) \chi_{\tau_{1}<\tau_{2} \wedge \theta}+ \\
+\phi_{2}\left(y\left(\tau_{2}\right)\right) \chi_{\tau_{2}<\tau_{1} \wedge \theta}+\phi_{0}(y(\theta)) \chi_{\tau_{2} \wedge \tau_{1}>\theta}
\end{array}
$$

Where in (2): $\chi_{E}$ is the caracteristic function of set $E, \theta(x)$ is the first time when the trajectory, solution of (1) with initial condition $x$ reachs $\partial \Omega(\theta(x)=\infty$ when the trajectory always remains in $\Omega$ ), that is:

$$
\theta(x)=\inf \{t: y(t) \notin \Omega\}
$$

$\phi_{0}$ is the final cost to pay if the game stops because the system leaves $\Omega, \mathrm{f}$ is the instantaneous cost, $\phi_{i}, i=1,2$ is the final costs if the game stops because player $i$ decides first to stop the game, with

$$
\phi_{2}(x) \geq \phi_{1}(x) \quad \forall x \in \Omega
$$

As the game is of zero sum, the goal of the first player is to maximize the payoff $\mathrm{J}$ and the goal of the second player is to minimize it. Our objective is to find (if they exist) $\bar{\tau}_{1}$ and $\bar{\tau}_{2}$ such that:

$$
V(x)=\sup _{\tau_{1}} \inf _{\tau_{2}} J\left(x, \tau_{1}, \tau_{2}\right)=J\left(x, \bar{\tau}_{1}, \bar{\tau}_{2}\right)
$$


where $\mathrm{V}$ is the value function of the game and $\bar{\tau}_{1}$ and $\bar{\tau}_{2}$ are optimal strategies or saddle points of the functional $\mathrm{J}$.

Inequality (3) indicates that the game is not a trivial game (no player can obtain advantages of priority of actions). It plays a key role in the proof of the existence of the value function but it does not represent a restriction as we can see in [20].

We suppose:

$$
(H) \begin{cases}g, & f, \quad \phi_{i}, \quad i=0,1,2 \text { are bounded functions in } \Omega \\ g, \quad f, \quad \phi_{i}, & i=0,1,2 \text { are Lipschitz continuous functions in } \Omega\end{cases}
$$

Remark 2.1 : We can see that condition (3) implies that the functional (2) is well defined (in this case finite strategies $\tau_{1}=\tau_{2}$ are not realistic strategies for both players).

Remark 2.2 : The limsup in (2) means that player 2 must minimize his worst situation.

Remark 2.3 : In definition (2), it is necessary to consider the limsup (or the liminf, in case we study the worst situation for player 1) in order to have a well defined payoff functional $J$. The fact that a limit need not exist can be easily seen in the following example. Let $x=\left(x_{1}, x_{2}\right) ; \phi_{2}(x)=M$ and $\phi_{1}(x)=-M$, with $M$ large enough such that $\tau_{1}=\tau_{2}=+\infty, f(x)=x_{1}, \Omega=(-2,2)$ and

$$
\frac{d x_{1}}{d t}(t)=x_{2}(t) ; \quad x_{1}(0)=1, \quad \frac{d x_{2}}{d t}(t)=-x_{1}(t) ; \quad x_{2}(0)=0
$$

in this case $\int_{0}^{\infty} f(y(s)) d s$ does not exist.

\subsection{Properties of the value function.}

We can define the lower and upper value of the game $\underline{V}$ and $\bar{V}$ respectively, in the following way:

$$
\underline{V}(x)=\sup _{\tau_{1}} \inf _{\tau_{2}} J\left(x, \tau_{1}, \tau_{2}\right) \quad \bar{V}(x)=\inf _{\tau_{2}} \sup _{\tau_{1}} J\left(x, \tau_{1}, \tau_{2}\right)
$$

If $(\mathrm{H})$ and (3) are valid we can prove, following the techniques used in [20], that $\underline{V}=\bar{V}=V . V$ will be the unique value function of the game. 
Remark 2.4 : In general, in terms of the value function $\epsilon$-optimal stopping times $\bar{\tau}_{1}^{\epsilon}, \bar{\tau}_{2}^{\epsilon}$ can be found, (see [10]), i.e. $\bar{\tau}_{1}^{\epsilon}, \bar{\tau}_{2}^{\epsilon}$, such that:

$$
J\left(x, \tau_{1}, \bar{\tau}_{2}^{\epsilon}\right)-\epsilon \leq J\left(x, \bar{\tau}_{1}^{\epsilon}, \bar{\tau}_{2}^{\epsilon}\right) \leq J\left(x, \bar{\tau}_{1}^{\epsilon}, \tau_{2}\right)+\epsilon \quad \forall \tau_{1}, \tau_{2}
$$

They are defined in the following way (see [11]):

$$
\bar{\tau}_{1}^{\epsilon}=\inf \left\{s / V(y(s)) \leq \phi_{1}(y(s))+\epsilon\right\} \quad \bar{\tau}_{2}^{\epsilon}=\inf \left\{s / V(y(s)) \geq \phi_{2}(y(s))-\epsilon\right\}
$$

Remark 2.5 : $V$ can be a discontinuous function as we can see in the following example.

Let $\Omega=[0, \pi]$ and the dynamic of the system given by: $\frac{d y}{d t}(t)=y(t), y(0)=x$, $f(x)=10 \sin x, \phi_{0}=0, \phi_{1}(x)=2 x-1, \phi_{2}(x)=2 x+1 ;$ then:

$$
V(x)=\left\{\begin{array}{lll}
\phi_{2}(x) & \text { if } & x \neq 0 \\
0 & \text { if } & x=0
\end{array}\right.
$$

\section{Isaacs inequality. Multiplicity of solutions.}

By the dymanic progamming principle we obtain that function $\mathrm{V}$ satisfies the following Isaacs inequation:

$$
\begin{gathered}
\phi_{1}(x) \leq V(x) \leq \phi_{2}(x) \quad \forall x \in \Omega \\
V(x)<\phi_{2}(x) \Rightarrow \exists \quad \delta_{x}>0 \quad / \quad \forall \delta<\delta_{x}, \quad V(x) \geq \int_{0}^{\delta} f(y(s)) d s+V(y(\delta)) \\
V(x)>\phi_{1}(x) \Rightarrow \exists \quad \delta_{x}>0 \quad / \quad \forall \delta<\delta_{x}, \quad V(x) \leq \int_{0}^{\delta} f(y(s)) d s+V(y(\delta))
\end{gathered}
$$

then for $\delta_{x}$ small enough we can inssure that $\mathrm{V}(\mathrm{x})$ is the fixed point of the following operator:

$$
V(x)=\left(M_{\delta_{x}} V\right)(x)=P_{I}\left\{\int_{0}^{\delta_{x}} f(y(s)) d s+V(y(\delta))\right\}
$$

where $P_{I}$ denotes the projection on interval $\mathrm{I}=\left[\phi_{1}(x), \phi_{2}(x)\right]$ i.e:

$$
P_{I}(a)= \begin{cases}\phi_{1}(x) & \text { if } a \leq \phi_{1}(x) \\ \phi_{2}(x) & \text { if } a \geq \phi_{2}(x) \\ a & \text { everywhere else }\end{cases}
$$


If we consider $x$ fixed and $y_{x}$ (t) the solution of (1) with initial condition $x$; we have that (4) has infinite solutions (manifold of one-dimension). Indeed, we define the following equivalence relation:

$x \sim x^{\prime}$ if there exists $t_{1} \geq 0$ such that $y_{x}\left(t_{1}\right)=x^{\prime}$ or $y_{x^{\prime}}\left(t_{1}\right)=x$.

Let $C_{x}$ be the equivalence classes and define $\varphi: C_{x} \rightarrow \mathbb{R}$; then: if $V($.$) is a solution$ of $(4), \bar{V}(x)=V(x)+\varphi\left(C_{x}\right)$ is also a solution if $\bar{V}(x) \in\left[\phi_{1}(x), \phi_{2}(x)\right]$.

Remark 2.6 : Properties of problems with boundary.

In order to avoid discontinuous solutions, we analyze the case where the following condition holds (see [11] and [19]):

$$
\begin{gathered}
\theta(x)<+\infty \quad \forall x \\
\exists a>0, \gamma>0 \quad / \quad d(y(s), \partial \Omega) \leq a \Rightarrow \frac{d}{d s} d(y(s), \partial \Omega) \leq-\gamma
\end{gathered}
$$

If (5) is valid we can prove that there exists a unique Lipschitz continuous value function $V$ and that there exists optimal strategies (see [10], [11]). This property arises because the exit time is a Lipschitz continuous function of $x$.

Remark 2.7 : Properties of some ergodic processes.

It is possible to give a precise characterization of the solution of our problem for a process such that the system remains in a compact manifold. In this case the solution of (1) defines in $\Omega$ a semigroup of translations $S(t)$. If we assume that this semigroup has a unique invariant measure that satisfies:

$$
\lim _{\tau \rightarrow \infty} \frac{1}{\tau} \int_{0}^{\tau} f\left(y_{x}(s)\right) d s=\int_{\Omega} f(z) d(\mu(z))=:<f, \mu>
$$

we say that the process is ergodic. Moreover if we suppose

$$
\int_{\Omega} f(z) d(\mu(z)) \neq 0
$$

then

$$
\lim _{\tau \rightarrow \infty} \int_{0}^{\tau} f\left(y_{x}(s)\right) d s=\infty \quad \operatorname{sign}\left\{\int_{\Omega} f(z) d(\mu(z)\}\right.
$$

We can prove that this condition implies the value function is uniformely Lipschitz continuous and that there exists optimal strategies.

$\operatorname{RR~} \mathrm{n}^{\circ} 2294$ 
Remark 2.8 : Characterization of the set where $V$ is a discontinuous function. Define, $\tau(x)=\bar{\tau}_{1} \wedge \bar{\tau}_{2} \wedge \theta$ if there exists finite optimal strategies; $\tau(x)=+\infty \wedge \theta$ if there does not exist optimal strategies; $A=\{x \in \Omega$ : $V(x)$ is discontinuous $\}$ and $\Omega_{\tau}=$ $\{x \in \Omega \quad: \quad \tau(x) \leq \tau\}$.

Then, it is easy to see that:

$$
A \subset \overline{\left(\bigcup_{\tau>0} \Omega_{\tau}\right)^{c}} .
$$

In order to obtain a numerical solution of the original problem we analize in the following two sections a time discretization and a space discretization (using finite element techniques, (see [7], [13], [14], [15], [16] and [21]).

\section{Time discretization.}

\subsection{The problem with discrete time}

To obtain a time discretization (see [7] for control problems and [1] for games problems), we consider $h>0$, an uniform partition of $[0,+\infty)$ in intervals of same length $\mathrm{h}$ and Euler approximation for the dynamics. Then, the Isaacs equation for time discretization becomes in this case:

$$
V^{h}(x)=\left(M^{h} V^{h}\right)(x)=\left\{\begin{array}{lll}
P_{I}\left[h f(x)+V^{h}(x+h g(x))\right] & \text { if } & x+h g(x) \in \Omega \\
P_{I}\left[\phi_{0}(x)\right] & \text { if } & x+h g(x) \notin \Omega
\end{array}\right.
$$

It is easy to see that $V^{h} \in B(\Omega)$ (the set of bounded functions in $\Omega$ ).

We can define a game problem with discrete stopping time associated to our real problem. The discrete time value function has the following form:

$$
\begin{aligned}
J^{h}\left(x, \tau_{1}, \tau_{2}\right)=\varlimsup_{\nu \rightarrow \infty} \sum_{\nu=1}^{\tau_{1} \wedge \tau_{2} \wedge \nu h \wedge \theta} f\left(y_{x}^{h}(\nu)\right)+\phi_{1}\left(y\left(\tau_{1}\right)\right) \chi_{\tau_{1}<\tau_{2} \wedge \theta}+ \\
+\phi_{2}\left(y\left(\tau_{2}\right)\right) \chi_{\tau_{2}<\tau_{1} \wedge \theta}+\phi_{0}(y(\theta)) \chi_{\tau_{2} \wedge \tau_{1}>\theta}
\end{aligned}
$$

where $\theta, \tau_{1}, \tau_{2} \in h Z^{+}$and the discrete dynamic is:

$$
y_{x}^{h}(0)=x ; \quad y_{x}^{h}(\nu+1)=y_{x}^{h}(\nu)+h g\left(y_{x}^{h}(\nu)\right)
$$

For sake of simplicity we denote:

$$
y^{\nu}=y_{x}^{h}(\nu)=\left(1-\frac{t-\nu h}{h}\right) y_{\nu}+\frac{t-\nu h}{h} y_{\nu+1} \quad \text { if } \quad t \in[\nu h,(\nu+1) h)
$$


The time discrete value function is:

$$
V^{h}(x)=\inf _{\tau_{1}} \sup _{\tau_{2}} J^{h}\left(x, \tau_{1}, \tau_{2}\right)
$$

The following theorem characterizes the set of all solutions of (7).

Theorem 3.1 Equation (7) has at least one solution. Moreover:

i) $V^{h}$ given by (10) is one of them.

ii) The following iteration

$$
w^{n+1}=M^{h} w^{n}, \quad w^{0} \geq \phi_{2}
$$

converges to the maximal solution that we call $\bar{w}$.

iii) $w^{n+1}=M^{h} w^{n}, \quad w^{0} \leq \phi_{1}$ converges to the minimal solution, $\underline{w}$.

iv) If $w($.$) is a solution, C_{x}^{h}=\left\{x^{\prime}=y_{x}^{h}(t), t \geq 0\right\}, \phi: C_{x}^{h} \rightarrow \mathbb{R}$, then $\bar{w}(x)=$ $w(x)+\phi\left(C_{x}^{h}\right)$ is also a solution if $\bar{w}(x) \in\left[\phi_{1}(x), \phi_{2}(x)\right]$.

Proof: We are going to prove item ii) (the others are similar). With condition $w^{0} \geq$ $\phi_{2}$ the sequence $M^{h} w$ is monotonously decreasing. In effect $w^{1}=M^{h} w^{0} \leq \phi_{2} \leq w^{0}$ and as $P_{I}$ is a monotonous operator, we have $w^{n+1}=M^{h} w^{n} \leq M^{h} w^{n-1}=w^{n}$. Moreover the sequence is bounded from below by $-\left\|\phi_{1}\right\|$. Then $w^{n}$ converges to $\bar{w}$ when $n \rightarrow \infty$ and it is a solution of (7). Moreover it is the maximal solution because if $\hat{w}$ is a solution of (7) $\hat{w}(x) \leq \phi_{2}(x) \quad \forall x$ then $\hat{w}=M^{h} \hat{w} \leq \phi_{2} \leq w_{0}$ and $\hat{w} \leq\left(M^{h}\right)^{n} w_{0}=w^{n} \quad \forall n$, that implies $\hat{w} \leq \bar{w}$.

\section{Remark 3.1 : Boundary problems.}

For the time discretization of problem presented in Remark 2.6 we obtain that the discrete solution is:

$$
V^{h}(x)=P_{I}\left[\phi_{0}(x)\right] \quad \forall x \in \partial \Omega_{h}^{+}=\{x \in \Omega / x+h g(x) \notin \Omega\}
$$

Remark 3.2 : a) Ergodic case with non zero mean, $(<\mu, f>\neq 0)$.

In this case we can identify the unique time discrete solution of the ergodic problem presented in Remark 2.7 because it is easy to see that:

$$
\begin{gathered}
\langle\mu, f>\neq 0 \Longrightarrow \\
\exists \bar{n}(x) / V^{h}(x)=\sum_{n=0}^{\bar{n}-1} f\left(y^{n}\right)+V^{h}\left(y^{\bar{n}}\right) ; \quad V^{h}\left(y^{\bar{n}}\right)=\phi_{i}\left(y^{\bar{n}}\right) \quad \text { with } \quad i=1 \text { or } 2 .
\end{gathered}
$$

$\mathrm{RR} \mathrm{n}^{\circ} 2294$ 
This result and iv) of theorem (3.1) prove the unicity of the discrete solution, moreover it is given by the usual iteration:

$$
w^{n+1}=M^{h} w^{n}, \quad w^{0} \text { given }
$$

b) Ergodic case with zero mean, $(<\mu, f\rangle=0)$..

In this case, it is easy to see that:

$$
\text { If } \phi_{1}(x)<\sum_{n=0}^{\nu-1} f\left(y^{n}\right)+V^{h}\left(y^{\nu}\right)<\phi_{2}(x) \quad \forall \nu \quad \text { then } \quad \underline{\lim }_{\nu} V^{h}\left(y^{\nu}\right)=0 .
$$

\section{Space discretization}

\subsection{Elements of the discrete problem}

To define a fully discrete problem it is necessary to discretize set $\Omega$, space $B(\Omega)$ and operator (7).

We approach $\Omega$ with $\Omega_{k}=\bigcup_{j} S_{j}^{k}$, where $S_{j}^{k}$ is a finite set of simplices; that is, $\Omega_{k} \subset \mathbb{R}^{\mathrm{n}}$ is a polyhedron with $\max _{j}\left(\operatorname{diam} S_{j}^{k}\right)=k$.

We denote $X=\left\{x_{i}, i=1, \ldots, N\right\}$ the set of vertices of $\Omega_{k}$ (nodes), $N$ the cardinality of $X$ and $\partial \Omega_{k}^{+}=\left\{x_{i} \in X / x_{i}+h g\left(x_{i}\right) \notin \Omega_{k}\right\}$.

We consider as approximation of $B(\Omega)$ the set $W_{k}$ of functions $w: \Omega_{k} \rightarrow \mathbb{R}, w$ continuous in $\Omega_{k}$, with $\frac{\partial w}{\partial x}$ constant in the interior of each simplex of $\Omega_{k}$ (that is, $w$ is a linear finite element).

Then, by definition of $W_{k}$, to obtain a solution of the discrete problem it is only necessary to obtain a solution in $X$. With the same methodology used in [15] and [16] we obtain a formulation of the fully discrete problem; (problem $P_{k}^{h}$ ); that is, to find a fixed point of the operator $M^{k}$ by the transformation: $V(x) \rightarrow$ $P_{I}[h f(x)+V(x+h g(x))]$ trough the natural isomorfism $W_{k} \leftrightarrow \mathbb{R}^{\mathrm{N}} \cdot M^{k}: \mathbb{R}^{\mathrm{N}} \rightarrow$ $\mathbb{R}^{\mathrm{N}}$, with $M^{k} w\left(x_{i}\right)=P_{I}(P w+F)\left(x_{i}\right)$.

If we call $V_{k}^{h}$ a solution of the fully discrete problem we have:

$$
V_{k}^{h}(i)=P_{I}\left(P V_{k}^{h}+F\right)(i)
$$

where $P_{I}$ denotes the projection on interval $\left[\phi_{1}(i), \phi_{2}(i)\right] ; P$ is a matrix with components $P_{i, j}$ given by:

$$
\begin{cases}x_{i}+h g\left(x_{i}\right)=\sum_{j=1}^{N} P_{i, j} x_{j}, & \text { if } x_{i} \notin \partial \Omega_{k}^{+} ; x_{i} \in \Omega_{k} \\ P_{i, j}=0 & \text { if } x_{i} \in \partial \Omega_{k}^{+}\end{cases}
$$


and $F$ is a vector of components:

$$
F\left(x_{i}\right)= \begin{cases}h f\left(x_{i}\right) & \text { if } x_{i} \notin \partial \Omega_{k}^{+}, x_{i} \in \Omega_{k} \\ P_{I}\left[\phi_{0}\left(x_{i}\right)\right] & \text { if } x_{i} \in \partial \Omega_{k}^{+}\end{cases}
$$

Because $\|P\|=1$ the operator $M$ is not a contractive operator and in the general case we can not insure a unique solution of (11).

\section{Markov chain associated to the process.}

We consider a random process with finite states $y_{i}^{(\nu)}$, where $\nu$ is the time discrete variable, $\mathrm{i}$ the initial state and the probability evolution is given by the matrix $P$ defined in (12) $\left(P_{i, j}\right.$ is the probability transition from node $\mathrm{i}$ to node $\left.\mathbf{j}\right)$.

In this Markovian system we can define a stochastic game problem with stopping times where the functional cost becomes:

$J^{k}\left(i, \tau_{1}, \tau_{2}\right)=$

$\varlimsup_{\nu \rightarrow \infty} E\left(\sum_{\nu=1}^{\tau_{1} \wedge \tau_{2} \wedge \nu h \wedge \theta} F\left(y_{i}^{\nu}\right)+\phi_{1}\left(y\left(\tau_{1}\right)\right) \chi_{\tau_{1}<\tau_{2} \wedge \theta}+\phi_{2}\left(y\left(\tau_{2}\right)\right) \chi_{\tau_{2}<\tau_{1} \wedge \theta}+\phi_{0}(y(\theta)) \chi_{\tau_{2} \wedge \tau_{1}>\theta}\right)$

where, $\theta, \tau_{1}, \tau_{2} \in h Z^{+}$are the exit and stopping times associated to the process and $F$ is defined in (13). For this discrete process the value function is:

$$
V_{h}^{k}(i)=\inf _{\tau_{2}} \sup _{\tau_{1}} J^{k}\left(i, \tau_{1}, \tau_{2}\right)
$$

Theorem 4.1 Equation (11) has at least one solution. $V_{h}^{k}$, given by (14) is one of its solutions.

Equation (11) has in general more than one solution. We call $S$ the set of its solutions. We are going to characterize $S$ taking in mind the fundamental structure of the graph associated to the Markov chain related with the discrete problem. We are going to identify in this set of solution the solution which represent the probabilistic formulation of the original problem.

We introduce the following equivalence relation in the set of nodes:

$i \sim j$ if $i=j$ or if there exists $i_{1}, \ldots, i_{\nu}, \nu>1$ such that $P\left(i_{m}, i_{m+1}\right)>0 \forall m=$ $1, \ldots, \nu \quad i_{1}=i_{\nu}=i$ and there exists $0<k<\nu$ with $i_{k}=j$.

This equivalence relation defines equivalence classes, $C^{r}, r=1, \ldots, \hat{N}$. We are going to define an order relation between these classes. So we define $S^{\nu}, \nu=1, \ldots, \bar{\nu}$ such that:

$\mathrm{RR} \mathrm{n}^{\circ} 2294$ 
$C^{r} \in S^{1}$ if it does not exist $\hat{r} \neq r$ such that $P(i, j)>0$, for any $i \in C^{r}, j \in C^{\hat{r}}$. $C^{r} \in S^{\nu} \quad \nu>1$ if there exists $\hat{r} \neq r$ such that $P(i, j)>0, i \in C^{r}, j \in C^{\hat{r}}, C^{\hat{r}} \in S^{\nu-1}$. If $C^{r} \in S^{1}, r=1, \ldots, n_{1}$, we say that $C^{r}=: C_{1}^{r}$ is a final class. We call transitory all other class that is not contained in $S^{1}$ and with $H$ the set of transitory elements. If $C^{r} \in S^{\nu}$ we set $C^{r}=C_{\nu}^{r}$. Without loss of generality we order the classes of $S^{1}$ putting in the first position classes $C_{1}^{r}=\left\{x_{j}\right\}$ corresponding to points $x_{j} \in \partial \Omega_{k}^{+}$. If $C_{\nu}^{r} \in S^{\nu}$ we denote cardinality of $C_{\nu}^{r}=m_{\nu}^{r}$

The analysis of (11) can be restricted to the analysis in each final class $C_{1}^{r}$ (taking in account that values in transitory classes are a function of values in elements of $S^{1}$ ). In effect the following theorem holds:

Theorem 4.2 If $i \in C_{\nu}^{r} \nu>1$ there exists a function $Q: \mathbb{R}^{\mathrm{t}} \rightarrow \mathbb{R}$, with $t=$ $\cup_{r=1}^{n_{1}} m_{1}^{r}$, where $V_{k}^{h}(i)=Q\left(V_{k}^{h}(j) j \in C_{1}^{r}\right)$.

Proof: For each $C_{1}^{r} \in S^{1} \quad r=1, \ldots n_{1}$ we obtain $v_{1} \in M_{m_{1}^{r} x 1}$ with

$$
v_{1}(i)=P_{\left[\phi_{11}(i), \phi_{21}(i)\right]}\left(P_{1} V_{1}+F_{1}\right)(i)
$$

where $P_{1} \in M_{m_{1}^{r} x m_{1}^{r}}$ is the restriction of matrix $\mathrm{P}$ considering only its action in $C_{1}^{r} \in S^{1}$. In the same way $F_{1}, \phi_{11}, \phi_{21} \in M_{m_{1}^{r} x 1}$ are the restriction of $F, \phi_{1}$ and $\phi_{2}$ respectively.

To compute the solution for $i \in C_{\nu}^{r} \in S^{\nu}, \nu>1 r=1, \ldots, n_{\nu}$ we compute in each class $C_{\nu}^{r} \in S^{\nu}, v^{\nu} \in M_{m_{\nu}^{r} x 1}$,

$$
v_{\nu}(i)=P_{\left[\phi_{1 \nu}(i), \phi_{2 \nu}(i)\right]}\left(P_{\nu} V_{\nu}+\bar{F}_{\nu}\right)(i)
$$

where $P_{\nu} \in M_{m_{\nu}^{r} x m_{\nu}^{r}}$ is the restriction of $P$ considering only its action in $C_{\nu}^{r} \in S^{\nu}$, $F_{\nu}, \phi_{\nu}, \phi_{2 \nu} \in M_{m_{\nu}^{r} x 1}$, with $\bar{F}_{\nu}=\sum_{j=1}^{\nu-1} P_{j} v_{j}+F^{\nu}$.

By Theorem(4.2) we obtain:

Theorem 4.3 Let $v, w$ be solutions of $(11)$; if $v\left(x_{i}\right)=w\left(x_{i}\right) \forall x_{i} \in C_{1}^{r} \subset S^{1}$ then $v=w$

\section{Study of equation (11) in final classes}

1) Case $x_{j} \in \partial \Omega_{k}^{+}$. This case corresponds to the space discretization of time discrete problem presented in Remark 3.1. Here the solution is obviously:

$$
V_{k}^{h}\left(x_{j}\right)=P_{I}\left[\phi_{0}\left(x_{j}\right)\right]=\min \left[\phi_{2}\left(x_{j}\right), \max \left(\phi_{1}\left(x_{j}\right), \phi_{0}\left(x_{j}\right)\right)\right] .
$$


2) Case $x_{j} \notin \partial \Omega_{k}^{+}$. We consider the restriction of matrix $P$ to the final class $C_{1}^{r}$. Without loss of clarity we denote also by $P$ this matrix. By definition of final class, we know that $P$ is an irreducible stochastic matrix. We call $d$ the greatest common divisor of the length of all the circuits (closed path) determinated by $P$. We define the following equivalence relation:

$$
i \sim j \Longleftrightarrow \exists \text { a path between } \mathrm{i} \text { and } \mathrm{j} \text { with a length multiple of } \mathrm{d}
$$

We denote $c_{i}, i=1, \ldots, d$, the $d$ subclasses of equivalence. Matrix $P$ has the following structure:

$$
P=\left[\begin{array}{llll}
0 & P^{1,2} & 0 & 0 \\
0 & 0 & P^{2,3} & 0 \\
0 & 0 & 0 & P^{d-1, d} \\
P^{d, 1} & 0 & 0 & 0
\end{array}\right]
$$

where we denote $P^{i, j}=P^{c_{i}, c_{j}}$. Matrix $P^{d}$ define an ergodic Markov chain in each subclass $c_{i}$, then (see [18]) there exists a unique invariant measure $\mu^{c_{i}}=\mu^{i}$ of $\left(P^{d}\right)^{i, i}$, that is, $\mu^{i}\left(P^{d}\right)^{i, i}=\mu^{i} \quad \forall i=1, \ldots, d$.

These invariant measures are related each other in the following way:

$$
\mu^{i} P^{i, i+1}=\mu^{i+1} \quad \forall i=1, \ldots, d
$$

Then $\mu=\frac{1}{d}\left(\mu^{1}, \ldots, \mu^{d}\right)$ is the unique invariant measure of the Markov process $\mathrm{P}$, i.e., $\mu=\mu P$.

Remark 4.1 : Measure $\mu$ determines the characterization of the solutions of (11).

Case $<\mu, f>\neq 0$

Theorem 4.4 If $\langle\mu, f\rangle \neq 0$ then the solution of (11) is unique and it is given by the limit point of the following convergent iteration,

$$
v^{\nu+1}=M^{k} v^{\nu} ; \quad \forall v^{0} \text { initial }
$$

Outline of the proof: In this case we can proof that there exists $x_{i} \in X$ such that $V\left(x_{i}\right)=\phi_{j}\left(x_{i}\right)$ with $j=1$ or 2 and then as we can inssure that the game finishes in a finite time we can prove that there exists $\hat{n}$ such that $\left(M^{k}\right)^{\hat{n}}$ is a contractive operator.

Remark 4.2 : To find numerically the solution of (17) we can use an adaptation of the accelerated algorithms presented in [9] and in [17]. 
Case $\langle\mu, f>=0$

In this case (11) can have more than one solution.

First, to prove the existence of solution of (11) (always restricted to a final class) we are going to study the solution of the linear system: $w\left(x_{i}\right)=(P w+F)\left(x_{i}\right)$.

Let $F=\left(\left(F^{1}\right)^{t}, \ldots,\left(F^{d}\right)^{t}\right)^{t} ; F^{i}=F^{c_{i}}$ (where $F^{t}$ means the transpose matrix), we are going to analyze the asymptotic behavior of functions:

$$
\phi_{n}(i)=E\left(\sum_{j=1}^{n} F\left(y_{j}\right)\right)=\left(\sum_{j=1}^{n}\left(P^{j-1} F\right)(i)\right)
$$

Sequence $\phi_{n}(i)$ has the following properties

$$
\begin{gathered}
\text { i) } \phi_{m d}(i) \rightarrow \eta_{0}(i) \quad \forall i \quad \text { if } m \rightarrow+\infty \\
i i) \phi_{m d+j}(i) \rightarrow \eta_{j}(i) \quad \forall i \quad \text { if } m \rightarrow+\infty
\end{gathered}
$$

Proof of i)

We have:

$$
\phi_{m d}=\sum_{q=1}^{d} P^{q-1} F+P^{d} \phi_{(m-1) d}=\hat{F}+P^{d} \phi_{(m-1) d}
$$

where $\hat{F}=\sum_{j=1}^{d-1} P^{j} F$.

As $P^{d}$ is block diagonal and each block defines a finite ergodic Markov chain we have that (19) geometrically converges to a finite value. Then there exists $\eta_{0}(i) \forall i$. ii) is obvious by i) and by the relation $\phi_{m d+j}=F+P \phi_{m d+j-1}$

Theorem 4.5 Characterization of solutions. The set of solution of the linear system $\phi=P \phi+F$ is a one-dimensional manifold.

Computation of a discrete solution of $\quad \phi=P \phi+F$

We want to compute $\overline{\lim }_{n \rightarrow+\infty} \phi_{n}=\max _{j} \eta_{j}(i)=\bar{\eta}(i)$ because it represents the solution of the linear system we want.

As $P^{m d} \rightarrow G$ when $m \rightarrow+\infty$, (where $G=\sum_{j} \hat{e}^{j} \hat{\mu}^{j}, \hat{e}^{j}=(0, \ldots, 1, \ldots, 1,0 \ldots, 0)$ with 1 in the $j$-th cycle and $\hat{\mu}^{j}=\left(0, \ldots, \mu^{j}, \ldots, 0\right)$ with $\mu^{j}$ the invariant measure vector restrict to subclass $c_{j}$ ).

By (18) and the properties of sequence $\phi_{n}$ we have $\eta_{j}$ satisfies the following equation:

$$
\eta_{j}=\eta_{0}+\left(\sum_{r=1}^{d} \hat{e}^{r} \hat{\mu}^{r}\right)\left(\sum_{q=1}^{j-1} P^{q} F\right)=\eta_{0}+\sum_{r=1}^{d} \hat{e}^{r} \sum_{q=1}^{j-1} \hat{\mu}^{r+q} F
$$


We have, in each element of the cycle, calling $w_{\rho}=\hat{\mu}^{\rho} F$, that:

$$
\eta_{j}(s)=\eta_{0}(s)+\sum_{\rho=s}^{s+j-1} w_{\rho}
$$

Let $\bar{\rho}$ such that $\sum_{r=1}^{\bar{\rho}} w_{r}=\max _{\rho} \sum_{r=1}^{\rho} w_{r}$ then we obtain $\overline{l i m}_{n \rightarrow \infty} \phi_{n}(i)=\max _{j} \eta_{j}(i)=$ $\eta_{\bar{\rho}}(i) \quad \forall i$

Proposition $4.1<\mu^{j}, \eta_{0}^{j}>=0, i=1, \ldots, d$ where $\eta_{0}=\left(\left(\eta_{0}^{1}\right)^{t}, \ldots,\left(\eta_{0}^{d}\right)^{t}\right)^{t}$.

\section{Procedure to compute $\bar{\eta}$}

1. Consider $c_{p}$, compute $n_{p}=$ cardinality of $c_{p}$ and determine $\hat{p}$ such that $n_{\hat{p}}=$ $\min _{p} n_{p}$.

2. Compute the asymptotic probability $\mu^{\hat{p}}$ and recursively $\mu^{p}$ for all $p$ as a function of $\mu^{\bar{p}}$.

3. Compute $w_{\rho}=<\mu^{\rho}, F^{\rho}>$ and determine $\bar{\rho}$ such that $\sum_{r=1}^{\bar{\rho}} w_{r}=\max _{\rho} \sum_{r=1}^{\rho} w_{r}$.

4. Compute $\eta_{0}^{\bar{\rho}}$ trough equation: $\eta_{0}^{\bar{\rho}}=\left(P^{d}\right)^{\bar{\rho}}, \bar{\rho} \eta_{0}^{\bar{\rho}}+\tilde{F}^{\bar{\rho}}$ where $\tilde{F}^{\bar{\rho}}=\sum_{j=0}^{\bar{\rho}-1} P^{j} F$ with additional condition $\left.<\mu^{j}, \eta_{0}^{j}\right\rangle=0$.

5. Compute $\eta_{\bar{\rho}}(s)=\eta_{0}(s)+\sum_{\rho=s}^{s+\bar{\rho}-1} w_{\rho}$.

Accelerated Algorithm to compute the solution of (11)

We are going to present an algorithm to obtain the solution of (11). The procedure finds in a finite number of steps, $\overline{\bar{\eta}}$, the limit point of iterations $v^{\nu+1}=M^{k} v^{\nu}$, with $v^{0}=\bar{\eta}$, where $\bar{\eta}$ is the solution of $\bar{\eta}=P \bar{\eta}+F$ that not satisfies $\phi_{1} \leq \bar{\eta} \leq \phi_{2}$ (if $\bar{\eta}$ satisfies this condition it is just the solution of (11)). The methodology is an extension of the one presented in [9] to the case where operator $M^{k}$ is not a contractive operator. As a matter of fact, we denote $V$ the solution of our continuous problem and:

$$
\begin{gathered}
S_{1}=\left\{j \in X / V(j)=\phi_{1}(j)\right\} \\
S_{2}=\left\{j \in X / V(j)=\phi_{2}(j)\right\} \\
\tilde{S}_{2}^{0}=\left\{j / \bar{\eta}(j)-\phi_{2}(j)=\max _{i}\left(\bar{\eta}(i)-\phi_{2}(i)\right)\right\} \bigcap\left\{j / \bar{\eta}(j)>\phi_{2}(j)\right\} \\
\tilde{S}_{1}^{0}=\left\{j / \bar{\eta}(j)-\phi_{1}(j)=\max _{i}\left|\bar{\eta}(i)-\phi_{1}(i)\right|\right\} \bigcap\left\{j / \bar{\eta}(j)<\phi_{1}[(j)\}\right.
\end{gathered}
$$

Then, we can prove: 
Lemma 4.1 $\tilde{S}_{i}^{0} \subset S_{i}$ with $i=1$ or 2

Proof: Let $\tilde{S}_{2}^{0} \neq \emptyset$, we denote $\Delta=\max _{i}\left(\bar{\eta}(i)-\phi_{2}(i)\right)$, in the same way the vector with components equals to $\Delta$ and $\mathrm{P}$ the matrix defined in (11). Then, taking in mind that $P \Delta=\Delta$, we have:

$\bar{\eta}-\Delta \leq \phi_{2} \quad \forall j=1, \ldots, N$

$\bar{\eta}-\Delta=\phi_{2} \quad \forall j \in \tilde{S}_{2}^{0}$

$P(\bar{\eta}-\Delta)+F=P \bar{\eta}+F-P \Delta=\bar{\eta}-\Delta$

As $M^{k}$ is a monotone operator:

$$
\begin{gathered}
P_{\left[\phi_{1}, \phi_{2}\right]}(P(\bar{\eta}-\Delta)+F)=P_{\left[\phi_{1}, \phi_{2}\right]}(\bar{\eta}-\Delta) \geq \bar{\eta}-\Delta \\
M^{k}(\bar{\eta}-\Delta) \geq \bar{\eta}-\Delta
\end{gathered}
$$

by induction

$$
\left(M^{k}\right)^{m}(\bar{\eta}-\delta) \geq M^{k}(\bar{\eta}-\Delta) \geq \bar{\eta}-\Delta
$$

but

$$
\left(M^{k}\right)^{m}(\bar{\eta}-\Delta)(i) \rightarrow \bar{w}(i), \text { when } m \rightarrow \infty
$$

then

$$
\bar{w}(i) \geq\left(M^{k}\right)^{m}(\bar{\eta}-\Delta)(i) \geq\left(M^{k}\right)(\bar{\eta}-\Delta)(i) \geq(\bar{\eta}-\Delta)(i)=\phi_{2}(i) \quad \forall i \in \tilde{S}_{2}^{0}
$$

that implies $i \in S_{2}$. Analogously, we obtain $\tilde{S}_{1}^{0} \subset S_{1}$.

By Lemma (4.1) we can deduce that we know the solution in $\tilde{S}_{1}^{0} \cup \tilde{S}_{2}^{0}$. We are going to use this information to find the discrete solution. We redefine matrix $P$ and $F$ in the following way: $\quad P^{0}=P, F^{0}=F, w^{0}=\bar{\eta}$ and $\forall n \geq 1 w^{n}$ is the solution of

$$
w^{n}=P^{n} w^{n}+F^{n}
$$

where

$$
\begin{gathered}
P^{n}(i, j)= \begin{cases}0 & \forall i \in \tilde{S}_{1}^{n-1} \cup \tilde{S}_{2}^{n-1} \\
P^{n-1}(i, j) & \text { if not }\end{cases} \\
F^{n}(i)= \begin{cases}\phi_{1}(i) & \forall i \in \tilde{S}_{1}^{n-1} \\
\phi_{2}(i) & \forall i \in \tilde{S}_{2}^{n-1} \\
F^{n-1}(i) & \text { if not }\end{cases}
\end{gathered}
$$

with

$\tilde{S}_{2}^{n}=\left\{j / w^{n}(j)-\phi_{2}(j)=\max _{i}\left(w^{n}(i)-\phi_{2}(i)\right)\right\} \bigcap\left\{j \quad / \quad w^{n}(j)>\phi_{2}(j)\right\}$

$\tilde{S}_{1}^{n}=\left\{j \quad / \quad w^{n}(j)-\phi_{1}(j)=\max _{i}\left|w^{n}(i)-\phi_{1}(i)\right|\right\} \cap\left\{j \quad / \quad w^{n}(j)<\phi_{1}(j)\right\}$

$S_{2}^{n}=\bigcup_{m=0}^{n} \tilde{S}_{2}^{m} ; \quad S_{1}^{n}=\bigcup_{m=0}^{n} \tilde{S}_{1}^{m} ; \quad C^{n}=C\left(S_{1}^{n} \cup S_{2}^{n}\right)$. 
Remark 4.3 : We know that there exists unique solution of (20) because $P^{n}$ has a submatrix of norm smaller than 1 .

Lemma 4.2 It is valid:

$$
w^{n}(i)= \begin{cases}\phi_{1}(i) & \text { if } i \in \tilde{S}_{1}^{n-1} \\ \phi_{2}(i) & \text { if } i \in \tilde{S}_{2}^{n-1}\end{cases}
$$

The proof is obvious by definitons of $P^{n}$ and $F^{n}$ given in (20).

We consider the following operator $M_{n}^{k}(w)$ defined by:

$$
\left(M_{n}^{k} w\right)(j)=P_{I}\left(P^{n} w+F^{n}\right)(j)
$$

with $P^{n}$ and $F^{n}$ defined in (20). It is easy to see that our solution is also the fixed point of operator $M_{n}^{k}$ that is:

$$
\bar{w}=M_{n}^{k} \bar{w}
$$

The following Lemma holds:

Lemma $4.3 \tilde{S}_{1}^{n} \cup \tilde{S}_{2}^{n} \neq \emptyset$ or $w^{n}=\bar{w}$

Proof: If $\tilde{S}_{1}^{n} \cup \tilde{S}_{2}^{n} \neq \emptyset$ and if $i \notin S_{1}^{n-1} \cup S_{2}^{n-1}$, we have:

$$
w^{n}(i) \in\left[\phi_{1}(i), \phi_{2}(i)\right]
$$

then

$$
\left(M_{n}^{k} w^{n}\right)(i)=P_{I}\left(P^{n} w^{n}+F^{n}\right)(i)=P_{I}\left(w^{n}\right)(i)=w^{n}(i)
$$

so, by (21) and (22) we have:

$$
w^{n}=\bar{w}
$$

\section{ALGORITHM}

Step 0: $\quad \mathrm{n}=0$

Step 1: Compute $w^{n}$ as in (20). Obtain $\tilde{S}_{1}^{n}$ and $\tilde{S}_{2}^{n}$

Step 2: $\quad \tilde{S}_{1}^{n} \cup \tilde{S}_{2}^{n} \neq \emptyset$ then stop.

If not, obtain $P^{n}(i, j)$ and $F^{n}(i)$ defined in (20). Let $n=n+1$ and go to step 1 .

Theorem 4.6 The algorithm finishes in a finite number of steps smaller than $N$. 
Proof: $\tilde{S}_{1}^{n} \cup \tilde{S}_{2}^{n} \neq \emptyset$ implies cardinality of $C^{n}<$ cardinality $C^{n-1}$. But as cardinality of $C^{1} \leq N$, there exists an index $\tilde{n}$ such that cardinality of $C^{\tilde{n}}=\operatorname{cardinality} C^{\tilde{n}-1}$, then $\tilde{S_{1}^{n}} \cup \tilde{S}_{2}^{n}=\emptyset$ and by Lemma $(4.3) w^{\tilde{n}}=\tilde{w}$.

Remark 4.4 : We can modifiy this algorithm taking $w^{0}=\phi_{2}$ as the initial vector (see [9]).

\section{Structure of the set of solutions}

We are going to characterize the structure of all the solutions of (11) in final classes - In the last sections we have proved that we must take in account the following cases:

1. there exists $\bar{\eta}$ (the limit point of sequence $\phi_{n}$ ) such that $\phi_{1} \leq \bar{\eta} \leq \phi_{2}$. In this case $w=\bar{\eta}+c e$ is also solution if $w(x) \in\left[\phi_{1}(x), \phi_{2}(x)\right] \forall c \in[c m i n, c m a x]$ where $c, c \min , c \max$ are constants and $e$ the identity vector.

2. $\bar{\eta}$ does not satisfy the last condition, then the iterative algorithm

$$
v^{n+1}=M^{k} v^{n} ; \quad v^{0}=\bar{\eta}
$$

converges to the solution of problem (11) and the following situations can occur:

- $\overline{\bar{\eta}}$ is solution of the linear system, then there exists a constant $a>0$ such that $\overline{\bar{\eta}}+c e$ is a solution $\forall c \in[0, a]$ or $\forall c \in[-a, 0]$.

- $\overline{\bar{\eta}}$ is not solution of the linear system, then is the "unique" solution of (11).

\section{Convergence properties}

When we can inssure that the problem finishes in a finite time, we can easily prove the convergence property (of type $\sqrt{k}$ ) of the unique discrete solution of our problem. That is the case of boundary problems or ergodic problems with mean different to zero.

In this cases we can reduce the problem to a finite time problem (see [1], [2], [3], [4] and [5]) and we can prove the convergence, in the viscosity sense) with the techniques using in [3], [14], [15] and [16] for this type of problem. 


\section{Conclusion}

We have studied an undiscountied zero sum differential game problem with stopping time. In this case we loose in general the regularity properties of the value function. We have showed an easy example where the value function is discontinuous. This fact implies the difficulty of its numerically resolution because the discrete problem may have infinite numerical solutions and it might not converge.

We have totally characterized the set of solutions of the discrete problem. We have obtained the solution that represent the probabilistic formulation of the original problem and we have presented an algorithm to compute it.

In regular cases we have proved the convergence of the discrete problem to the real solution because in these cases the problem can be associated to a finite time problem and we can use usual techniques to prove convergence properties.

\section{References}

[1] Bardi M., Soravia P., " Approximation of differential games of pursuit-evasion by discrete-time games". Developments in modeling and computation, R.P. Hamalainen, H.K. Ethamo eds. Lectures Notes in Control and Information Sciences 156. Springer-Verlag, pp 131-143. 1991.

[2] Bardi M., Soravia P., " Hamilton-Jacobi equations with singular boundary conditions on a free boundary and applications to differential games". Trans. Amer. Math. Soc. 325. pp 205-229. 1991.

[3] Bardi M., Falcone M., Soravia P., " Fully discrete schemes for the value function of pursuit-evasions games". Proceedings of Fifth International Symposium on Dynamic Games and Applications, (Grimentz, Switzerland, 15-18 July 1992).

[4] Bardi M., Falcone M., " Discrete approximations of the minimal time function for systems with regular optimal trajectories". Ricerca di Atenes. Ottimizzatione de Funcionali e Convergence Variazionali. 1990.

[5] Bardi M., Falcone M., "An approximation schemes for the minimum time function". Siam J. Control and Optimization. Vol 4 pp 950-965. 1990.

[6] Barles G., Perthame B., " Exit time problems in optimal control and vanishing viscosity method." Siam J. Control and Optimization, Vol 26, No 5, pp 1133, 11481988. 
[7] Capuzzo Dolcetta I., Ishii H., " Approximate solution of the Bellman equation of deterministic control theory". Appl. Math. Optim., Vol. 11, pp. 161-181, 1984.

[8] Crandall M. G., Lions P. L., "Viscosity solutions of Hamilton-Jacobi equations". Tansactions of the American Mathematical Society, Vol 277, No. 1, pp. 1-42, 1983.

[9] Di Marco S., "Técnicas de descomposición-agregación en el tratamiento de la inecuación bilátera de Isaacs". Mecánica Computacional Vol 12, pp 509-518, 1991.

[10] Friedman A., " Differential Games". (Wiley-Interscience, New York, 1971).

[11] Gonzalez R., " Sur la resolution de l'equation de Hamilton-Jacobi du côntrole deterministique". Cahiers de Mathématiques de la Decisión N 8029 and 8029 bis. Ceremade-Université de Paris-Dauphine, 1980.

[12] Gonzalez R., " Solución numérica de problemas de juegos diferenciales de suma nula con tiempo de detención". Anales del $1^{e} r$ Congreso Nacional de Informática y Teleinformática -USUARIA'83/13 JAIIO, pp. 4.1-4.17, (Buenos Aires, 1983).

[13] Gonzalez R., Rofman E., "On deterministic control problems: an approximation procedure for the optimal cost. Part I and II". SIAM Journal on Control and Optimization, 23, pp. 242-285, 1985.

[14] Gonzalez R., Tidball M., " On a Discrete Time Approximation of the Hamilton-Jacobi Equation of Dynamic Programming," Rapport de Recherche N1375, INRIA, 1990.

[15] Gonzalez R., Tidball M., " On the rate of convergence of fully discrete solutions of Hamilton-Jacobi equations ". , Rapport de Recherche , N 1376, INRIA, 1991.

[16] Gonzalez R., Tidball M., "Sur l'odre de convergence des solutions discrétisées en temps et en espace de l'equation de Hamilton-Jacobi". Comptes Rendus Acd. Sci., Paris, Tomo 314, Serie I, pp. 479-482, 1992.

[17] Gonzalez R. L. V., Tidball M. M., " Fast solution of general nonlinear fixed point problems". System Modeling and Optimization, Proceedings of the 15th 
IFIP Conference on System Modeling and Optimization, Zurich, Switzerland, (September 2-6, 1991), Lecture Notes in Control and Information Sciences, Vol.180, pp. 35-44, (Springer Verlag, New York, 1992).

[18] Ross S. M., " Applied Probability Models with Optimization Applications" . (Holden-Day, San Francisco, 1970).

[19] Soner M., "Optimal control with state-space constraints, II". SIAM J. of Control and Optim., 26, pp. 1110-1122, 1986.

[20] Stettner L., " Zero-sum Markov Games with Stopping and Impulsive Strategies". Appl. Math. Optim., Vol. 9, pp. 1-24, 1982.

[21] Strang G., Fix G., " An Analysis of the Finite Element Method". (PrenticeHall, Englewood Cliffs, NJ, 1973).

[22] Tidball M.M, Gonzalez R.L.V., " Zero sum differential games with stopping times. Some results about its numerical solution". Proceedings of Fifth International Symposium on Dynamic Games and Applications, (Grimentz, Switzerland, 15-18 July 1992). Annals of Dynamics Games, Vol 1, 1993. 
Unité de recherche INRIA Lorraine, Technopôle de Nancy-Brabois, Campus scientifique, 615 rue du Jardin Botanique, BP 101, 54600 VILLERS LĖS NANCY

Unité de recherche INRIA Rennes, Irisa, Campus universitaire de Beaulieu, 35042 RENNES Cedex

Unité de recherche INRIA Rhône-Alpes, 46 avenue Félix Viallet, 38031 GRENOBLE Cedex 1

Unité de recherche INRIA Rocquencourt, Domaine de Voluceau, Rocquencourt, BP 105, 78153 LE CHESNAY Cedex

Unité de recherche INRIA Sophia-Antipolis, 2004 route des Lucioles, BP 93, 06902 SOPHIA-ANTIPOLIS Cedex

Éditeur

INRIA, Domaine de Voluceau, Rocquencourt, BP 105, 78153 LE CHESNAY Cedex (France)

ISSN 0249-6399 